\title{
1 New class of graphene-based devices for the next generation of nonvolatile memories
}

\subsection{Introduction}

Graphene is a one-atom thick layer of carbon atoms arranged in a hexagonal lattice. Graphene potentialities are attracting a lot of researchers to probe opportunities in a number of directions in the "more Moore" or "beyond CMOS" optics in order to identify the new future technologies [1-3]. Another promising utilization of graphene and related nanomaterials is to fabricate nonvolatile memories (NVM) exploiting their "memresistive" behavior storing a value of electrical resistance in a permanent way. This happens when a current passing through the materials changes the level of resistance. Therefore, resistive memory exploits the change in the resistance of a material under the effect of an electric field as an information write/erase principle for nonvolatile data storage. The reading of resistance states is nondestructive, and the memory devices can be operated without transistors in every cell [4, 5], as for flashtype memories [6-10] (see Section 1.2.2), thus achieving a classic cross-bar structure. This kind of memories is called resistive random-access memory (RRAM or ReRAM) and is only one of the possible types of nonvolatile ways to store information in a permanent way. One of the most important advantages of these new classes of $2 \mathrm{D}$ materials is that these materials can be implemented in flexible electronics [11-15], in the form of one-thick atom layers as for graphene or in the form of layers of flakes of graphene oxide (GO) or reduced GO (R-GO), thereby reducing the final cost of the final device exploiting roll-to-roll fabrication [16]. Another great advantage of ReRAMs is their potential to implement them by exploiting only two terminals to work (two contacts and not three as a common transistor, which has drain, source and gate, e.g., flash-type memories), which could dramatically reduce the circuitry and allow to implement easily in 3D architectures by using the roll-to-roll fabrication technique. Potentially, this is applied in various fields such as health monitoring [17-21], intelligent packaging [22, 23], cards, labels, badges, value paper and medical disposables. Another potential field is the radiofrequency identification (RFID). This is a technology to electronically record the presence of an object using radio signals. Indeed, an innovative alternative pathway to reduce RFID costs and integrate a memory chip to store data is to eliminate the silicon substrate completely, and produce RFID and memory on the same flexible plastic substrate as the antenna [24-26].

Paolo Bondavalli, Thales Research and Technology, Palaiseau Cedex, France 
Thanks to graphitic layers, the antenna and chip can be built on the same low-cost substrate, and attachment costs can be removed.

\subsection{Graphene-based NVM}

As outlined in the International Technology Roadmap for Semiconductors $2011 \mathrm{sec}$ tion concerning Emerging Research Devices, "ultrathin graphite layers are interesting materials for macromolecular memories thanks to the potential fabrication costs that are considered as the primary driver for this type of memory, while extreme scaling is de-emphasized." The main drawback is related to the fact that memory operation mechanisms and the physics are still unclear and that a deeper research in this field is necessary to improve the comprehension of the phenomenon and the efficiency of the devices. These are not the same physical mechanisms exploited in memories based on graphene-related materials, which will be discussed in the next paragraphs.

\subsubsection{Graphene and graphitic layers}

The first paper highlighting the utilization of graphene for resistive NVM exploiting two terminal structures was issued in 2008 by Stadley et al. at Caltech [27]. They reported the development of an NVM element based on graphene break junctions. These junctions were obtained by achieving two-terminal devices, transferring graphene sheets on $\mathrm{SiO}_{2} / \mathrm{Si}$ substrate and depositing metal electrodes on them using e-beam lithography process. After applying a specific voltage under ultravacuum condition $\left(10^{-7}\right.$ torr), they were able to create a break in the graphene layer (see Figure 1.1). In these papers, all the measurements were performed under ultravacuum to avoid environmental gas interferences, which strongly reduces the potential utilization of the device.

After cycling the memories, they observed that the resistance values clearly changed as a function of the voltage (see Figure 1.1b): they moved from a high resistance state (off state) to a lower one as a function of the voltage (on state). This effect was nonvolatile and the devices were cycled thousands of writing cycles $\left(10^{5}\right)$, showing long retention times (more than $24 \mathrm{~h}$, only limited by measurements).

The reproducibility of the switching behavior was very promising. One of the more coherent explanations was related to the fact that the conductance occurred along a small graphene ribbon that bridges the contacts. To better understand the underlying physical mechanism, researchers studied the time-resolved behavior of the switch from the OFF to ON states, shown in Figure 1.2a. The conductance $I / V$ showed well-defined steps, with magnitude $\sim G_{Q}$. Here $G_{Q}$ is $\mathbf{2 e}^{\mathbf{2}} / \mathbf{h}$, the conductance quantum, where $\boldsymbol{e}$ is the 

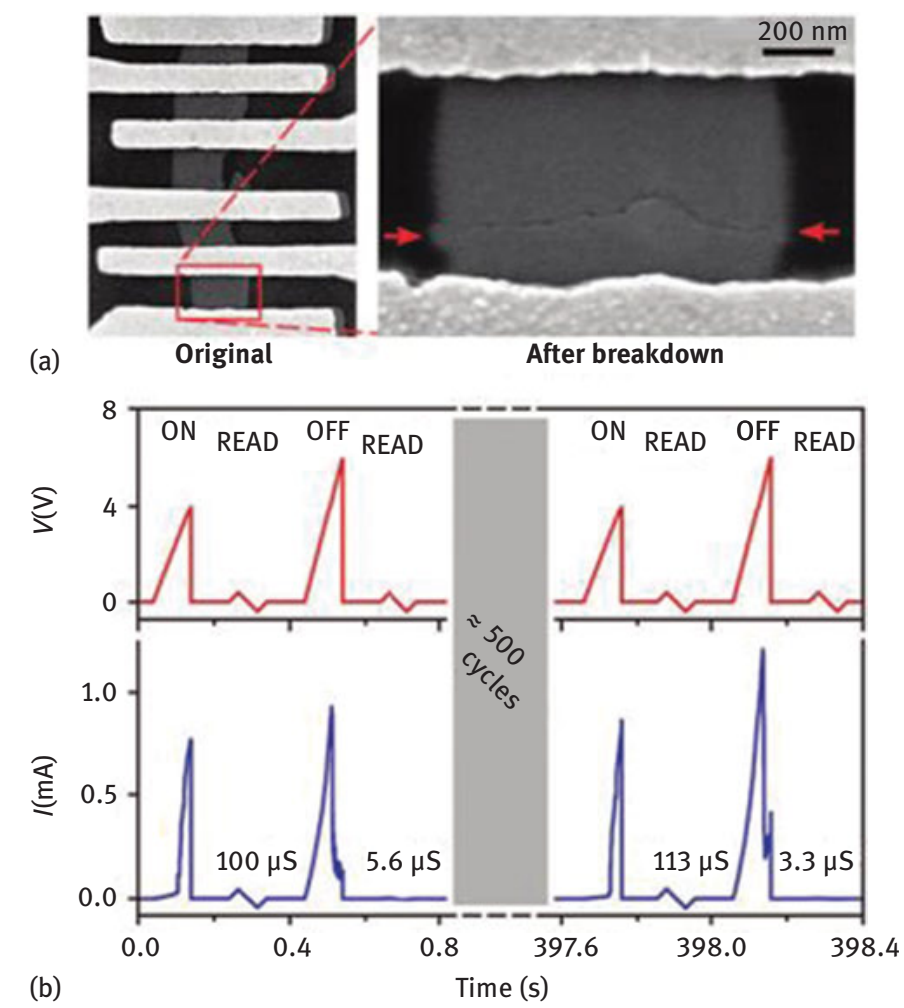

Figure 1.1: (a) SEM image of the device before (left panel) and after breakdown (right panel).

The arrows indicate the edges of the break in the graphene layer. (b) Repeatable programming over hundreds of cycles. Upper left panel: Voltage applied to the junction versus time. A ramp with a peak value of $\sim 4 \mathrm{~V}$ corresponds to an ON pulse, while a ramp with a peak value of $\sim 6 \mathrm{~V}$ corresponds to an OFF pulse. A small sawtooth-shaped read-out pulse is applied after each write to determine the junction conductance. Lower left panel: Current flow through the junction, with the low-bias conductance labeled above each read-out pulse. Right panels: Similar data taken after approximately 500 intervening cycles, demonstrating the reproducibility of the switching behavior [27].

electron charge and $\boldsymbol{h}$ is Planck's constant. Since $G_{\mathrm{Q}}$ is the conductance of a spindegenerate one-dimensional conductor, for example, a linear chain of gold atoms [28], observation of steps in the conductance suggests that the conductance states of a device are likely multiples of highly transmitting quantum channels. Authors proposed a model for device operation based on the formation and breaking of carbon atomic chains that bridge the junctions (see Figure 1.2a, right figure). This mode and the results seem to put in evidence the potential for multiresistive states.

The same physical explanation can be evoked for the results obtained in 2009 by Sinitski et al. [29] at Rice when they tested analogue carbon-based structures (see Figure 1.2b). The sensitive layer was consists of $10 \mathrm{~nm}$ thick graphitic disks 

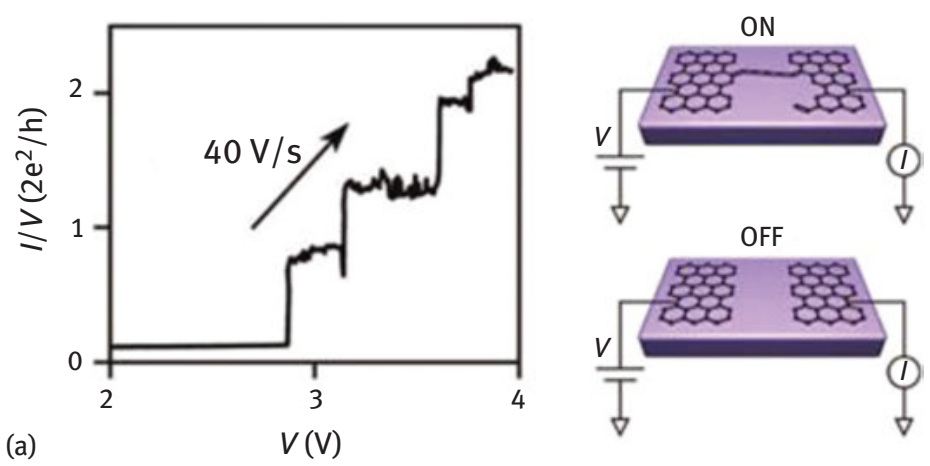

(a)

$V(\mathrm{~V})$

(b)
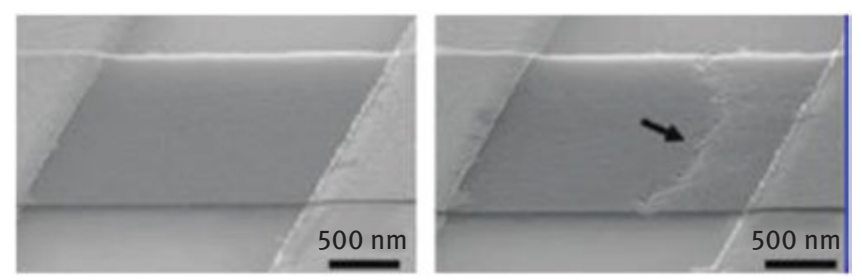

Figure 1.2: (a) Left side: Change in $I / V$ as a function of $V$ pointing out the quantic behavior linked to the formation of atomic chains. Right side: Proposed physical mechanism at the base of the nonvolatile resistive behavior (formation of atomic carbon chains). (b) Tilted-view SEM images of two different devices before and after applying the voltage difference that is necessary to break the layers defined as $V_{\text {break. }}$. The arrow shows the fracture across the stripe due to $V_{\text {break }}$ [29].

grown by chemical vapor deposition (CVD) process. Researchers evaluated the voltage that is necessary to break the layer. Calculating the Joule heating, researcher obtained that the breakdown voltage, the applied voltage difference necessary to break the layer, was

$$
\mathrm{V}_{\text {break }} \alpha\left[\left(\mathrm{C} \rho \gamma \mathrm{T}_{\text {break }}\right) / \tau\right]^{1 / 2} 1=\mathrm{A}
$$

where $\mathrm{C}, \rho, \gamma$ are, respectively, the specific heat, the density of CVD-grown graphitic sheets and resistivity, $\boldsymbol{T}_{\text {break }}$ is the breakdown temperature of the graphitic layer, $\boldsymbol{A}$ is independent of the devices and $\boldsymbol{l}$ the length of the device. Therefore, the voltage necessary to break the layer is directly proportional to the length. This is very positive because reducing the dimensions will allow to reduce the energy consumption during the writing step.

In 2009, the same team from the University of Rice [30] exploited another approach and grew graphitic layers on a freestanding silicon oxide nanowire (see Figure 1.3a). Indeed they used the nanowire to preserve the mechanical integrity of the device after applying a specific voltage breaking the surficial graphitic layers. The team from Rice fabricated two terminal devices consisting of discontinuous 


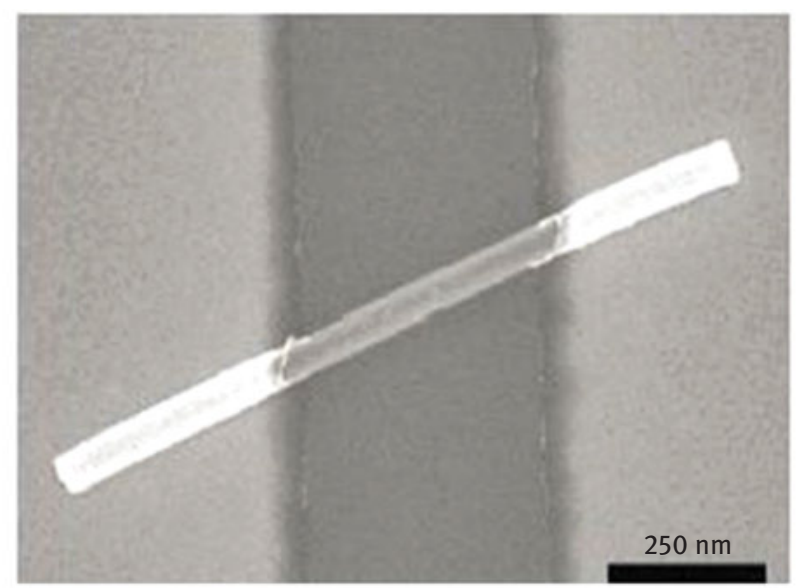

(a)

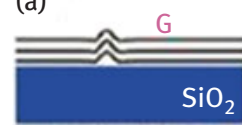

As - fabricated

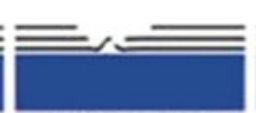

After opening

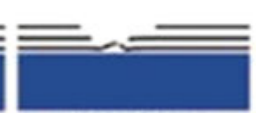

After writing

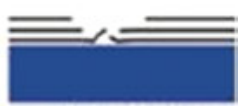

After erasing

(b)

Figure 1.3: (a) Silicon oxide nanowire with graphitic layers grown on its surface and (b) physical principle at the base of the fabrication of nonvolatile graphitic memories exploited by Rice University [30].

5-10 nm thin films of graphitic sheets grown by CVD on nanowires having stable, rewritable, nonvolatile and nondestructive read memories with on/off ratios of up to $10^{7}$ (see Figure 1.4a) and switching times of up to $1 \mu$ s (tested limit).

All the samples were not tested, as in the previous examples, at ambient conditions but in ultra-vacuum condition $\left(5 \times 10^{-5}\right.$ torr) and showed an extremely good stability of the performances after several days and also after x-ray exposure (Figure 1.4b).

An interesting work by Wu et al. [31] in 2012 dealt with the electrical properties of graphene sheet suspended on a patterned indium tin oxide (ITO) electrode pair. They measured very interesting on/off ratio of 6 orders and retention time of at less $10^{4} \mathrm{~s}$ in ambient conditions. In this case, data storage was achieved by applying voltage bias and rewritten after a simple heat treatment. Authors stated that the switch happens only in ambient atmosphere when oxygen changed the injection barrier at the contact. The degassing using a thermal process allowed coming back to the high conduction state. 

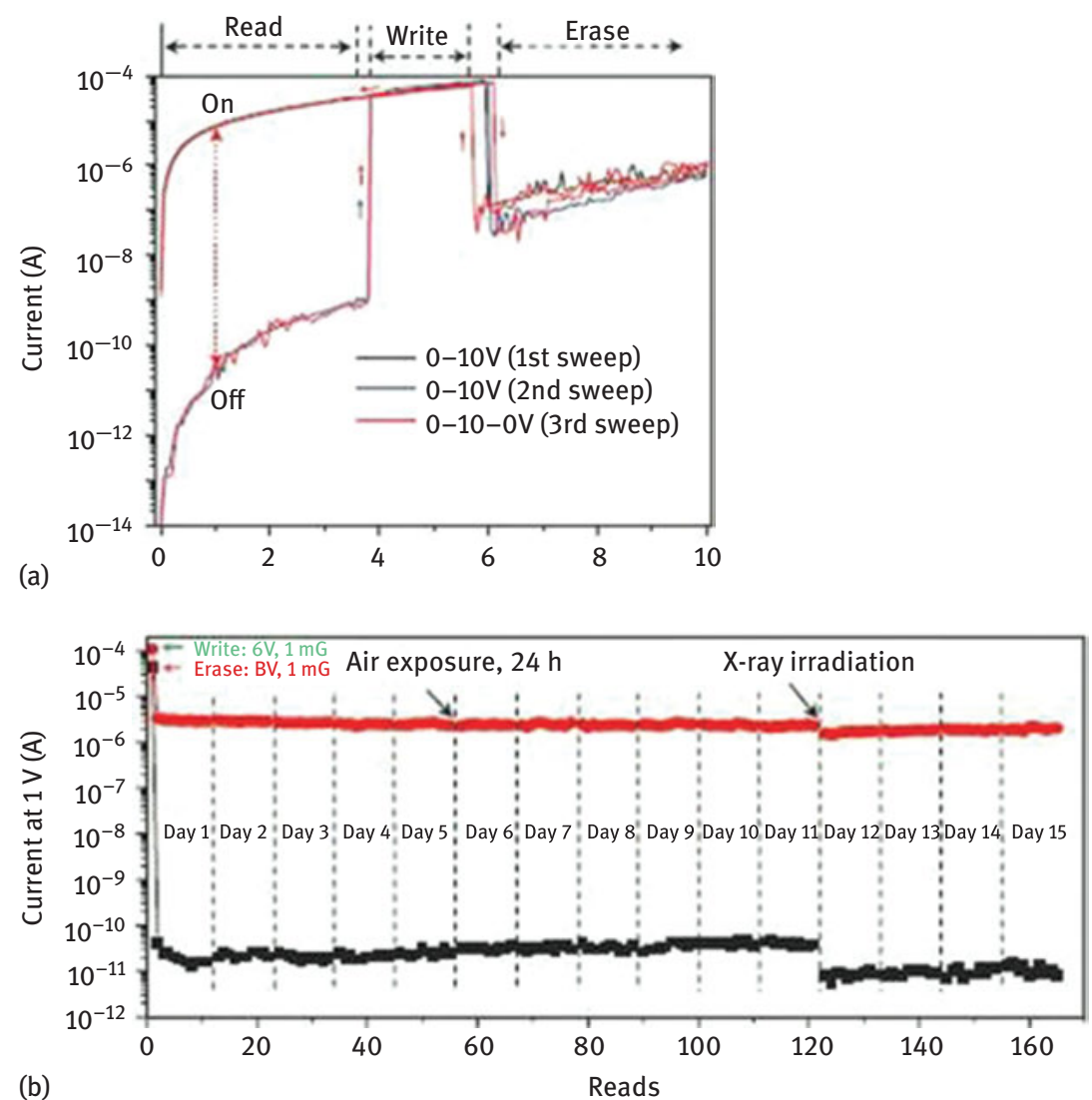

Figure 1.4: (a) The logarithmic $I-V$ behavior of a $\mathrm{G}-\mathrm{SiO}_{2}$ nanoribbon; the "write" or "erase" regions can be achieved through a voltage pulse in the noted regions. After writing or erasing, the high- or low-conductivity state of the system is recorded in the "read." (b) Data retention by two other $\mathrm{G}-\mathrm{SiO}_{2}$ nanocable devices [30].

\subsubsection{Nonvolatile resistive memories based on GO and R-GO oxide layers}

Other teams focused their study on GO. GO is a wide band gap material $(6 \mathrm{eV})$ with potential for modulation, thanks to oxidation/reduction process providing tunability of the electronics, mechanical and optical properties. GO [32] is commonly obtained by the oxidation of graphite using the modified Hummer's method, where the long oxidation time is combined with a highly effective method for the purification of reaction products $[33,34]$. A great advantage of this material is its cost, which is at least one-tenth the price of graphene flakes and it is stable in water suspensions. In GO, the memory configuration exploited is similar to cross-bar memories 


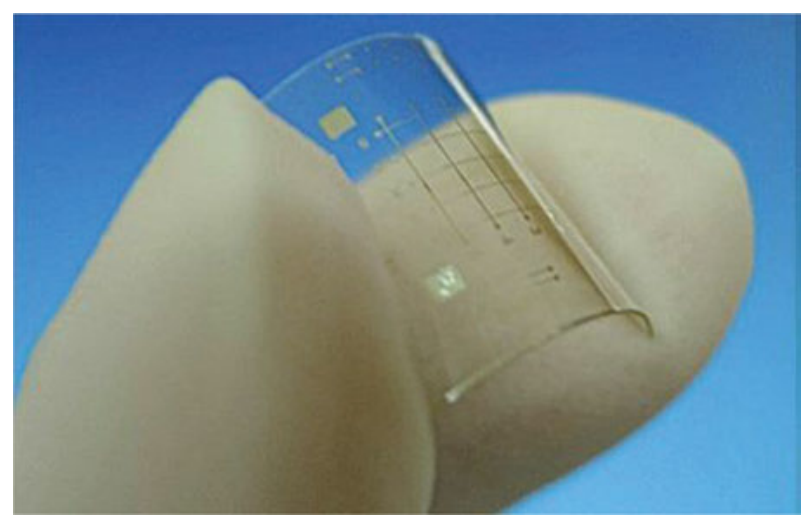

Figure 1.5: Cross-bar memory devices based on $\mathrm{GO}[39,40]$.

$[35,36]$ (vertical junctions composed by piling up the different layers, sandwiching the active one as metal insulator metal-type structures). We can mention the pioneering work of $\mathrm{He}$ et al. in 2009 [37], where reliable and reproducible resistive switching behaviors were observed in $\mathrm{GO}$ thin films prepared by vacuum filtration method [38]. They fabricated $\mathrm{Cu} / \mathrm{GO} / \mathrm{Pt}$ structures showing an on/off ratio of about 20 , a retention time of more than $10^{4} \mathrm{~s}$ and switching threshold voltages of less than $1 \mathrm{~V}$. The suggested physical principle at the origin of the switching effect was the desorption/absorption of oxygen-related groups on the GO sheets as well as the diffusion of the top electrodes. One of the major works on GO-based memories was issued in 2010 by Jeong et al. [39, 40]. This team demonstrated the nonvolatile effect on the resistance of a $70 \mathrm{~nm}$ layer thick $\mathrm{GO}$ in a layered structure composed of $\mathrm{Al} / \mathrm{GO} / \mathrm{Al}$ (Figure 1.5) in a cross-bar configuration.

Jeong et al. were one of the first teams that pointed out the performances of these kinds of layers on flexible substrates. The researchers from KAIST demonstrated that using GO layers they were able to achieve flexible nonvolatile resistive memories with impressive performances under traction and compression [they kept three orders between on (LRS) and off (HRS) levels]. Concerning the physics explanation of the phenomenon, Hong et al. [41, 42] performed a deep analysis of the switching mechanism for this kind of devices and demonstrated that these structures had performances dependent on the origin of the top contacts. For example, in case of Au-based top electrodes, there was no oxygen migration in opposition to Al electrodes. The effect of bottom contacts was also quite important. Indeed if the roughness was too high, the GO layer had such cracks and rough surface that the top electrode material could easily penetrate and then build filaments that hindered the switching operations. This drastically reduced the lifetime of the device (only 100 cycles) because of the formation of a permanent conductive path between the two electrodes. These results were highlighted by X-ray photoelectron spectroscopy measurements that pointed 
out the permanent presence of $\mathrm{Al}$ near the bottom electrode as the main reason for failure. Evaluating the effect of the migration of oxygen versus the formation of Albased filaments, Hong and coworkers stated that the formation of conducting filaments was a local phenomenon and that the oxygen migration was the dominant mechanism. Indeed, they discovered that the leakage current between the bottom and lower electrodes was dependent on the cell dimension. In fact when the cells were larger the effect of the oxygen migration or reduction on the conduction was enhanced proportionally to the surface. In order to avoid the failure mechanism linked to the formation of permanent conductive filaments through the material cracks, one suggestion is to exploit deposition techniques that allow a more uniform distribution of the deposited material. One technique is the deposition by spray coating or heating the substrate to avoid the so-called coffee-ring effect $[43,44]$, which is responsible for the nonuniformity of the deposition [45]. This deposition could potentially prevent the formation of cracks linked to the roughness in the GO layers.

Another interesting study was performed by Vasu et al. at the University of Bangalore (India) in 2011 [46]. They reported very simple unipolar resistive switching device using ultrathin $(\sim 20 \mathrm{~nm})$ films of R-GO with on/off ratios reaching five orders of magnitude. The thin films were formed at room temperature simply by drop-casting R-GO from suspension on ITO glass electrode, followed by aluminum or gold deposition (no difference was pointed out after the measurements). A very interesting result was also the switching time that could attain $10 \mu$ sith an on/off ratio of 100. In this case, the formation of nanofilaments of carbon atoms was evoked as the main physical working principle.

\subsection{Other approaches to achieve nonvolatile memories using graphitic layers}

In the previous sections, we have discussed about the resistive NVM. Other teams have decided to use different approaches to exploit the properties of graphenebased layers to fabricate NVM and have exploited the hysteretic electrical behavior of graphene, or multilayered graphene (MLG), when used as channel in a field effect transistor (FET)-type configuration. This is substantially based on the chemical modification of graphene under an electric field that will enhance environmental interactions. However, the more promising results, in terms of performances but also in terms of potential industrial exploitation, have been obtained in case of the fabrication of flash-type memory [47-48] using graphene or MLG as the floating gate (FG). Basically, a flash memory is an electronic nonvolatile computer storage medium that can be electrically erased and reprogrammed. In flash memory, each cell resembles a standard MOSFET [85], where the transistor has two gates instead of one. Indeed there is the traditional top gate, also called control gate and an FG 
embedded in an oxide layer; hence, it is electrically insulated. In FG, which is located between the top gate and the MOSFET channel, any electrons placed on it, activating the top gate, are trapped there and will not discharge for many years. If FG holds a charge, it screens (partially cancels) the electric field from the top gate; therefore, it modifies the threshold voltage $\left(\mathrm{V}_{\mathrm{T}}\right)$ of the MOSFET. This operation is nonvolatile and is reversible if another adequate voltage is applied on the top gate. The present technology used for flash-type memories employs doped polysilicon as material for the FG. One of the main advantages of graphene/MLG is the reduction of voltage to achieve a correct memory window, which is the shift of the threshold voltage of the transistor when the memory is switched from 0 to 1 state. In the present flash memories, a voltage difference of $\pm 20 \mathrm{~V}$ [49] is necessary to program/erase to achieve a memory window of $1.5 \mathrm{~V}$, which is the industrial standard. Some works have demonstrated, as shown in the next paragraphs that thanks to the higher density of states (DOS) of graphene compared to degenerately doped polysilicon this difference can reduced up to 6 Volts. Another advantage is the higher work function of graphene [50-52] that is directly linked to the larger barrier height between the FG and the oxide [53-56], which embeds the FG. This potentially allows achieving longer retention time, potentially more than ten years. Finally, the possibility to achieve device using 2D devices with a lower dimensionality that will potentially allow an easier 3D implementation and so a higher memory density.

\subsubsection{Graphitic-based nonvolatile memory using a transistor configuration}

One of the first paper highlighting the interest of using graphene-based FET for NVM was issued by Wang et al. at the Nanyang Technological University in Singapore in 2010 [57]. Indeed they observed exactly the same phenomenon observed in case of FET with one carbon nanotube as a channel: unhysteretic phenomenon where they observed the formation of a loop of the current value between drain and source, $\mathrm{I}_{\mathrm{ds}}$, as a function of the gate voltage, $\mathrm{V}_{\mathrm{gs}}$, during the voltage sweep cycle from negative to positive values of $\mathrm{V}_{\mathrm{gs}}$ and back. This phenomenon is caused by the presence of moisture interacting with the transistor dielectrics. This effect, it has been demonstrated, can be removed in vacuum $[58,59]$. Moreover the switching time in case of activation of the hysteretic phenomenon in FET using graphene is of the order of some seconds, which is too high for memory applications.

In 2008, a pioneering work of Echtermeyer et al. of the Advanced Research Center in Aachen (Germany) and of A. K. Geim at the University of Manchester (UK) was performed adopting another point of view compared to the previous studies $[60,61]$. Considering the difficulty to achieve nanoribbons with dimensions smaller than $10 \mathrm{~nm}$ to open a gap, they exploited another phenomenon. Indeed they 
fabricated two-gate transistor structures as shown in Figure 1.6, where the graphene has a width of $10 \mu \mathrm{m}$ and was covered by an $\mathrm{SiO}_{2}$ layer.

(a)

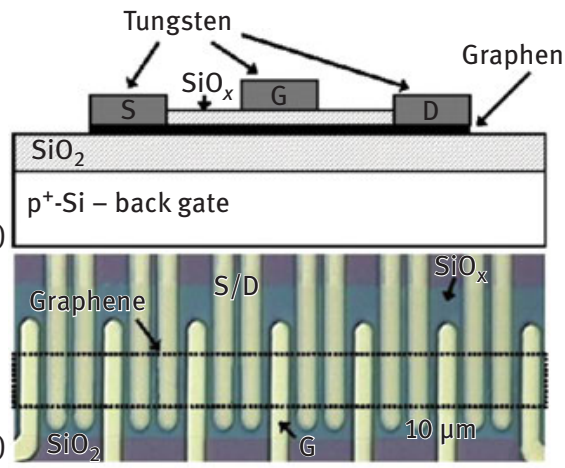

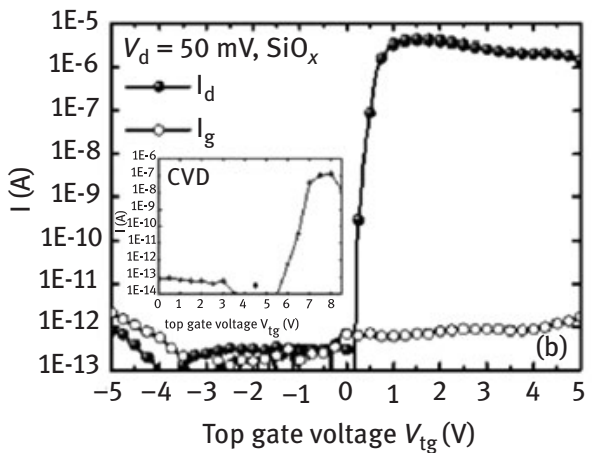

Figure 1.6: (a) Schematic of a double-gated graphene field effect device (FED) and (b) current between drain and source as a function of the top gate voltage value $[60,61]$.

They observed that applying a voltage spanning from -5 to $+5 \mathrm{~V}$, for positive voltage there was a strong enhancement in the current of 5/6 order, hence, a reduction in the resistance that was nonvolatile. Echtermeyer and coworkers suggested that considering that the measurements had been performed in ambient conditions, the water molecules were split in $\mathrm{H}^{+}$and $\mathrm{OH}^{-}$(see Figure 1.6) by the electric field and tended to attach to the graphene, creating graphene or GO (that are insulating materials), passing through the highly porous silicon dioxide layer surface, and so drastically changing the conductivity of the channel in a nonvolatile way. This phenomenon allowed obtaining a strong on/off ratio, quite unusual for graphene (and not graphene nanoribbons) based FET. However, even if the results seem to be very promising, more studies have to be done on the switching time, which intuitively appears to be quite long considering the effects involved. We can add that the graphene layer has been obtained by mechanical cleavage, which is a technique that allows fabricating high-quality samples but that it is not industrially suitable. It is necessary to test this approach, for example, using CVD graphene in order to achieve a potential parallel fabrication of this kind of devices. It has also to be verified if it is fundamental to obtain a single graphene layer or if more stacked layers of graphene can reach the same performances. 


\subsubsection{Nonvolatile flash-type memories based on graphene/ multilayered graphene}

Another potential utilization of graphene is FG in flash-type memories. An extremely innovative approach has been proposed by Bertolazzi et al. of the Ecole Polytechnique de Lausanne (EPFL) in Switzerland. They were able to fabricate a flash memory using exactly the same architecture of the existing ones, exploiting only 2D materials [62].

Bertolazzi and coworkers were able to build a flash-type memory using $\mathrm{MoS}_{2}$ as the channel and few graphene layers as the $\mathrm{FG}$ (see Figure 1.7), embedded in $\mathrm{HfO}_{2}$ oxide, where the charge is trapped. It is clear that this kind of structure is extremely original but some performances especially considering the switching time (the lowest value is $100 \mathrm{~ms}$ ) are not adequate. Some doubts can be raised on the fabrication technique, which includes three quite complex transfer steps and that are not suitable at the moment for an industrial exploitation. Hong et al. at IBM in Watson
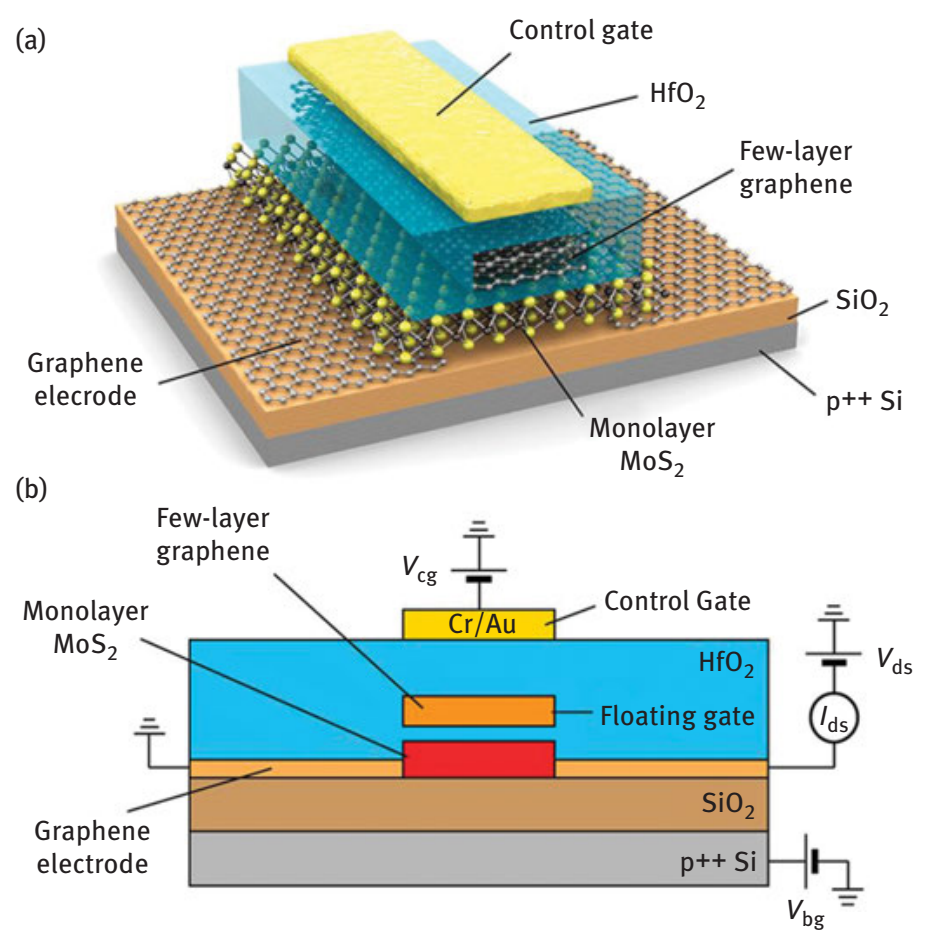

Figure 1.7: $\mathrm{MoS}_{2}$ /graphene heterostructure memory layout. (a) Three-dimensional schematic view of the memory device based on single-layer $\mathrm{MoS}_{2}$. (b) Schematic view of the flash-type memory cell with a single-layer $\mathrm{MoS}_{2}$ acting as a semiconducting channel and graphene contacts and multilayer graphene (MLG) as the FG [62]. 
(USA) fabricated a flash memory [63] using some graphitic layers, grown by CVD on $\mathrm{Si}$ as FG. In this case, the technological approach appears potentially scalable, CMOS compatible and so industrially suitable (see Figure 1.8) compared to the previous one. IBM researchers observed a potential retention time of 10 years, with a loss of only $8 \%$ of the charges in the FLG but also simulating the potential crosstalk between neighbor cells, who stated that this kind of memories showed negligible interference down to $10 \mathrm{~nm}$ (in case of common polysilicon FG, the interference raises dramatically under $25 \mathrm{~nm}$ ). Briefly, considering the power reduction (as told previously, thanks to the higher DOS compared to common FG fabricated using polysilicon) and the increase in the storage density, Hong and coworkers estimated a potential reduction of $75 \%$ in the operating energy of this kind of memory. However, in this work there are no data on the switching time and therefore we cannot compare their performances with existing memories.

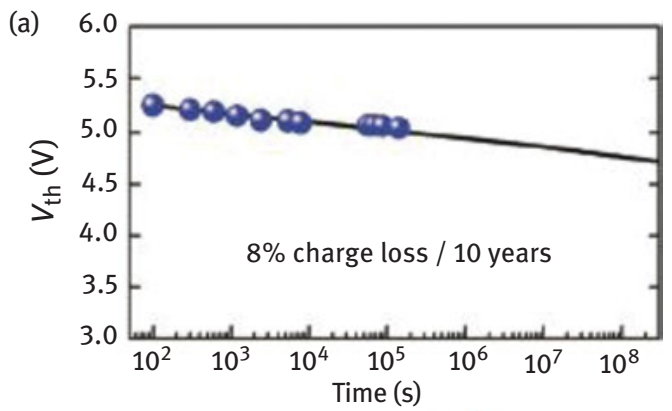

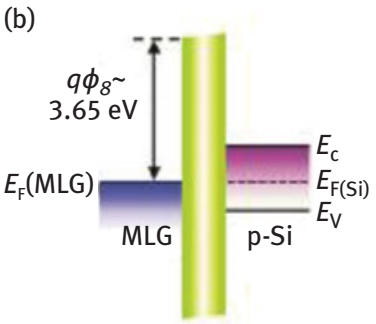

$\mathrm{SiO}_{2}$

(c) $\mathrm{SE}$

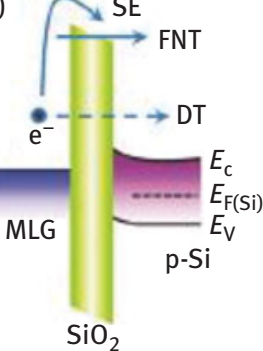

Before program $\left(V_{\mathrm{g}}=0\right)$ After program $\left(V_{\mathrm{g}}=0\right)$

Figure 1.8: Retention characteristics of GFM. (a) Retention measurement of MLG-FM showing only $8 \%$ of charge loss in 10 years at room temperature. (b) Energy band diagram of $\mathrm{MLG} / \mathrm{SiO}_{2} / \mathrm{Si}$ junctions before programming. (a) Energy band diagram after programming, which shows three possible mechanisms for charge loss during retention state: SE, FNT and DT. All three mechanisms depend exponentially on the barrier height $(\varphi B)$ between the work function of graphene and the $\mathrm{SiO}_{2}$ tunnel oxide [63]. 


\subsection{Conclusions and potential applications}

Graphene-based memories is a completely new technology that needs some time to demonstrate its utility especially in the difficult field of NVM for space application. These memories could constitute a real breakthrough compared to existing technologies also considering the dramatic potential reduction of energy consumption. These memories can be developed to bear harsh environments with a focus on radiationresistant components. The stability (also in temperature), resilience, nonvolatility and on/off ratio that, in certain cases, can attain $10^{7}$ or the switching time of $1 \mu$ s (competitive with flash-type memories), potential switching voltages of 3-4 V, combined with predictable fabrication-controlled $\mathrm{I}-\mathrm{V}$ behavior, simple two-terminal geometry (no need for a gate and so dramatically reducing circuitry) and access to mass fabrication for the two approaches make them extremely attractive structures for NVM.

Indeed, thanks to thin-film technologies, electronic functionality can be foreseen in very large quantity and at very low cost on substrates such as plastic and paper. Additional functionality is also an attractive feature of carbon-based memories. For example, by using these kinds of materials, we open up the route for memories on flexible substrates, a key building block to enable the success of flexible electronics. Carbon-based resistive memories should also offer the capability for multilevel storage and "memristive-like" behavior, as seen in other resistive memory materials. Multilevel storage allows storing more than one bit per cell, so increasing data storage densities, while memristive-like behavior can be exploited to provide a remarkable range of signal processing/computing-type operations, including implementing logic, providing synaptic and neuron-like "mimics," and performing, in a very efficient way, analogue signal processing functions (such as multiply accumulate operations).

In case of graphitic-based memories based on other approaches, the transistortype configuration exploiting the hysteretic effect, in our opinion, has a limited potential compared to new flash-type memories using graphene or MLG as the FG, because their architecture can be unlikely miniaturized and their performances especially considering the switching time are difficult to evaluate. In case of flashtype memories, these last exploit intrinsical characteristics of the materials such as the higher DOS (which allow reducing the voltage for the memory window) or the higher work function (which allows reaching more than 10 years of charge storage) that really allows improving the performances of this kind of memories. Moreover, it has been demonstrated to achieve high-density architecture with limited crosstalk. It is necessary to make an effort on the miniaturization of these devices but the recent works, for example, [51], make us think that this technology can be quite rapidly in competition with existing flash memories, also because it is CMOS compatible and easy to implement in existing production lines. 


\section{References}

[1] Ohishi, M. et al. Spin injection into a graphene thin film at room temperature. Jpn. J. Appl. Phys 2007, 46, L605-L607

[2] Cho, S., Chen, Y. F., Fuhrer, M. S. Gate-tunable graphene spin valve. Appl. Phys. Lett 2007, 91, 123-105.

[3] Lee, M. J., Suh, D. S., Seo, S., Jung, R., Ahn, S. E., Lee, C. B., Kang, B. S., Ahn, S. E., Lee, C. B., Seo, D. H., Cha, Y. K., Yoo, I. K., Kim, J. S., Park, B. H. Two series oxide resistors applicable to high speed and high density nonvolatile memory. Adv. Mater 2007, 19, (22) 3919-3923, 0935-9648.

[4] Waser, R., Aono, M. Nanoionics-based resistive switching memories. Nat. Mater. 2007, 6 (11) 833, 840, 1476-1122.

[5] Bez, R., Camerlenghi, E., Modelli, A., Visconti, A., Introduction to Flash memory. Proceeding of the IEEE. 2003, 91 (4), 489.

[6] Aritome, S., Advanced flash memory technology and trends for file storage application. Tech. Dig. IEDM 2000, 763.

[7] Muller, G., Happ, T., Kund, M., Yong Lee, G., Nagel, N., Sezi, R., Status and outlook of emerging nonvolatile memory technologies, In proceeding of: Electron Devices Meeting, 2004. IEDM Technical Digest. IEEE International.

[8] Lai, S., Flash memories: Where we were and where we are going. IEDM Tech. Dig. 1998, 971-973.

[9] Pavan, P., Bez, R., Olivo, P., and Zanoni, E., Flash memory cells - An overview. Proc. IEEE 1997, 85, 1248-1271, Aug.

[10] Pavan, P., Bez, R., The industry standard Flash memory cell, in Flash Memories, P. Cappelletti et al., Ed. Norwell, MA: Kluwer, 1999.

[11] Chao Yan, Jeong Ho Cho, Jong-Hyun Ahn, Graphene-based flexible and stretchable thin film transistors. Nanoscale 2012, 4, 48-70.

[12] Bae, S. , Kim, H., Lee, Y., Xu, X., Park, J.-S., Zheng, Y., Balakrishnan, J., Lei, T., Ri Kim, H., Song, Y. I., Kim, Y.-J., Kim, K. S., Ozyilmaz, B., Ahn, J.-H., Hong, B. H., lijima, S., Roll-to-roll production of 30-inch graphene films for transparent electrodes. Nat. Nanotechnol 2010, 5, 574-578.

[13] Wang, Y., Zheng, Y., Xu, X., Dubuisson, E., Bao, Q., Lu, J., Loh, K. P., Electrochemical delamination of CVD-grown graphene film: toward the recyclable use of copper catalyst. ACS Nano 2011, 5, 9927-9933.

[14] Lock, E. H., Baraket, M., Laskoski, M., Mulvaney, S. P., Lee, W. K., Sheehan, P. E., Hines, D. R., Robinson, J. T., Tosado, J., Fuhrer, M. S., Hernandez, S. C., Walton, S. G., High-quality uniform dry transfer of graphene to polymers. Nano Lett, 2011, 12, 102-107

[15] Xin, Wei, Liu, Zhi-Bo, Sheng, Qi-Wen, Feng, Ming, Huang, Li-Gang, Wang, Peng, Jiang, WenShuai, Xing, Fei, Liu, Yan-Ge, Tian, Jian-Guo, Flexible graphene saturable absorber on twolayer structure for tunable mode-locked soliton fiber laser. Optics Express, 2014, 22 (9) DOI:10.1364/OE.22.010239

[16] Secor, E. B., Prabhumirashi, P. L., Puntambekar, K, Geier, M. L., Hersam, M. C., Inkjet printing of high conductivity, flexible graphene patterns. J Phys Chem Lett, 2013, 4 (8), 1347-1351.

[17] Schwartz, G., Tee, B., Mei, J. et al. Flexible polymer transistors with high pressure sensitivity for application in electronic skin and health monitoring. Nat Commun 4, 1859 (2013). https://doi.org/10.1038/ncomms2832

[18] Sekitani, T., Zschieschang, U., Klauk, H., Someya, T. Flexible organic transistors and circuits with extreme bending stability. Nature Mater 2010, 9, 1015-1022.

[19] Ramuz, M., Tee, B. C.-K., Tok, J. B.-H., Bao, Z. Transparent, optical, pressure-sensitive artificial skin for large-area sretchable electronics. Adv Mater, 2012, 24, 3223-3227.

[20] Wagner, S. et al. Electronic skin: Architecture and components. Phys E, 2004, 25, 326-334. 
[21] Sokolov, A. N., Tee, B. C. K., Bettinger, C. J., Tok, J. B. H., Bao, Z. Chemical and engineering approaches to enable organic field-effect transistors for electronic skin applications. Acc. Chem. Res, 2012, 45, 361-371.

[22] Yam, L., Takhistov, Paul T., Miltz, Joseph, Intelligent packaging: Concepts and application kit. J Food Sci. 2005, 70, 1, pages R1-R10, January.

[23] Kololuoma, Terho K., Tuomikoski, Markus, Makela, Tapio, Heilmann, Jali, Haring, Tomi, Kallioinen, Jani, Hagberg, Juha, Kettunen, Ilkka Kopola, Harri K., Towards roll-to-roll fabrication of electronics, optics, and optoelectronics for smart and intelligent packaging. Proc. SPIE 2004, 77, 53-63, Emerging Optoelectronic Applications, (June 25).

[24] Kim, S., Cook, B., Le, T., Cooper, J., Lee, H., Lakafosis, V., Vyas, R., Moro, R., Bozzi, M., Georgiadis, A., Collado, A., Tentzeris, M M., Inkjet-printed antennas, sensors and circuits on paper substrate, IET microwaves, antennas \& propagation, 2013, 7 (10), 16 July, 858-868.

[25] Le, T., Ziyin Lin., Wong, C.P., Tentzeris, M.M., Novel enhancement techniques for ultra-highperformance conformal wireless sensors and "smart skins" utilizing inkjet-printed graphene. Electronic Components and Technology Conference (ECTC), 2013 IEEE 63rd, 2013, 1640, 1643, 28-31 May.

[26] Pham, H., Xiaochuan Xu, D.T., Chen, M. Y., Hosseini, A., Lu, Xuejun, Chen, R.T., Inkjet-printed two-dimensional phased-array antenna on a flexible substrate. Antennas and Wireless Propagation Letters, IEEE, 2013, 12, 170-173.

[27] Standley, B., Bao, W., Zhang, H. Bruck, J., Ning, Lau C., Bockrath, M., Graphene-based atomic-scale switches. Nanolett 2008, 8 (10), 3345-3349.

[28] Agrait, N., Levy-Yeyati, A., van Ruitenbeek, J. M. Quantum properties of atomic-sized conductor. Phys Reps 2003 377, 81-380.

[29] Sinitskii, A., Tour, J. M., Lithographic graphitic memories. ACS Nano 2009, 3 (9), 27-60.

[30] Li, Y., Sinitskii, A., Tour, J. M., Electronic two-terminal bistable graphitic memories. Nat Mat 2008, 7966.

[31] Wu, C., Li, F., Zhang, Y., Guo, T. Recoverable electrical transition in a single graphene sheet for application in nonvolatile memories. Appl Phys Lett 2012, 100, 042-105.

[32] Son, D. I., Kim, T. W., Shim, J. H., Jung, J. H., Lee, D. U., Lee, J. M., Park, W. I., Choi, W. K., Flexible organic bistable devices based on graphene embedded in an insulating poly(methyl methacrylate) polymer layer. Nano Lett 2010, 10, 2441-2447.

[33] Dreyer, Daniel R., Park, S., Bielawski, C. W., Ruoff, R. S., The chemistry of graphene oxide. Chem Soc Rev 2010, 39, 228-240.

[34] Hummers, W. S., Offeman, R. E. Preparation of graphitic oxide. J. Am. Chem. Soc 1958, 80, 1339.

[35] Snider, Greg, Kuekes, Philip, Williams, R Stanley, CMOS-like logic in defective, nanoscale crossbars. Nano Tech 2004, 15, 881-891.

[36] Linn, Eike, Rosezin, Roland, Kügeler, Carsten, Waser. Rainer, Complementary resistive switches for passive nanocrossbar memories. Nat Mater 2010, 9, 403-406.

[37] He, C. L., Zhuge, F., Zhou, X. F., Li, M., Zhou, G. C., Nonvolatile resistive switching in graphene oxide thin films. Appl Phys Lett 2009, 95, 232101

[38] Wu, Z., Chen, Z., Du, X., Logan, J. M., Sippel, J., Nikolou, M., Kamaras, K., Reynolds, J. R., Tanner, D. B., Hebard, A. F., Transparent, conductive carbon nanotube films. Science 2004, 305, 1273-1276.

[39] Jeong, H. Y., Yun Kim, O., Won Kim, J., Hwang, J. O., Kim, J-E., Yong Lee J., Yoon, T. H., Cho, B. J., Kim, S. O., Ruoff, R. S., Choi, S-Y. Graphene oxide thin films for flexible nonvolatile memory. Nanolett 2010, 10, 4381-4386.

[40] http://spectrum.ieee.org/semiconductors/nanotechnology/flexible-graphene-memristors.

[41] Hong, S. K., Kim, J. Y., Kim, J. W., Choi, S. Y., Cho, B. J., Flexible Resistive Switching Memory. Device Based on Graphene Oxide. IEEE Electron Device Lett 2010, 31, 1005. 
[42] Hong, S. K., Kim, J. E., Kim, S. O., Cho, B. J., Analysis on switching mechanism of graphene oxide resistive memory device. J Appl Phys 2010, 31 (1005), 2010

[43] Deegan, R. D., Bakajin, O., Dupont, T. F., Huber, G., Nagel, S. R. and Witten, T. A., Capillary flows as the cause of ring stains from dried liquid drops, Nature 1997, 1, 827.

[44] Deegan, R. D., Bakajin, O., Dupont, T. F., Huber, G., Nagel, S. R. and Witten, T. A., Contact line deposits in an evaporating drop. Phys Rev B 200062 (1), 756.

[45] Bondavalli, P, Delfaure, C., Legagneux, P., Pribat, D., Supercapacitor electrode based on mixtures of graphite and carbon nanotubes deposited using a new dynamic air-brush deposition technique. JECS 2013, 160 (4), A1-A6.

[46] Vasu, K. S., Sampath, S., Sood, A. K., Nonvolatile unipolar resistive switching in ultrathin films of graphene and carbon nanotubes. Solid State Communications 2011, 151 (16), 1084-1087

[47] Micheloni, Rino, Crippa, Luca, Marelli, Alessia, Inside NAND Flash Memories, Springer, e-book, ISBN 978-90-481-9431-5

[48] Masuoka, F., lizuka H., US4531203 (A) Semiconductor memory device and method for manufacturing the same.

[49] Masuoka, F., Momodomi, M., Iwata, Y., Shirota, R., New ultra high density EPROM and flash EEPROM with NAND structure cell. Electron Devices Meeting, 1987 International 1987, IEEE.

[50] Van Houdt, J., Degraeve, R., Groeseneken, G., Maes, H. E., Nonvolatile memory technologies with emphasis on flash: A comprehensive guide to understanding and using NVM devices, Published Online: 23 MAR 2007 DOI: 10.1002/9780470181355.ch4

[51] Kingon, Angus, Device physics: Memories are made of ..., Nature, 1999, 401, 658-659.

[52] Cheng Yuhua, Chenming Hu, MOSFET Classification and Operation. MOSFET Modeling \& BSIM3 User's Guide. Springer. 1999, 13, ISBN 0-7923-8575-6.

[53] Song, Seung Min., Park, Jong Kyung., Sul, One Jae., Cho, Byung Jin. Determination of work function of graphene under a metal electrode and its role in contact resistance. Nano Letters 2012, 12 (8), 3887-3892.

[54] Yu, Y., Zhao, Y., Ryu, S., Brus, L. E., Kim, K. S., Kim, P., Tuning the graphene work function by electric field effect. Nano Lett. 2009, 9, 3430-3434.

[55] Yan, L., Punckt, C., Aksay, I. A., Mertin, W., Bacher, G., Local voltage drop in a single functionalized graphene sheet characterized by Kelvin probe force microscopy. Nano Lett 2011, 11, 3543-3549.

[56] Filleter, T., Emtsev, K. V., Seyller Th., Bennewitz R., Local work function measurements of epitaxial graphene. Appl. Phys. Lett. 2008, 93, 133-117.

[57] Wang, Haomin., Wu, Yihong., Chunxiao, Cong., Jingzhi, Shang., Yu, Ting., Hysteresis of electronic transport in graphene transistors. ACS Nano 2010, 4 (12), 7221-7228. DOI:10.1021/nn101950n

[58] Kim, W., Javey, A., Vermesh, O., Wang, Q., Li, Y., Dai, H., Hysteresis caused by water molecules in carbon nanotube field-effect transistors. Nano Lett 2003, 3, 193-198.

[59] Bondavalli, P., Legagneux, P., Pribat, D., CNTFET based gas sensors: State of the art and critical review. Sensors Actuat B 140, 1, 304-318.

[60] Echtermeyer, T., Lemme, M., Baus, M., Szafranek, B., Geim, A., Kurz, H. Nonvolatile switching in graphene field-effect devices. IEEE Electron Device Lett. 2008, 29, 8, 952-954.

[61] Echtermeyer, T. J., Lemme, M. C., M. Baus, B., Szafranek, N., Geim, A. K., and Kurz, H., A graphene-based electrochemical switch. arXiv:0712.2026v1.

[62] Bertolazzi, S., Krasnozhon, D., Kis, A. Nonvolatile memory cells based on $\mathrm{MoS}_{2} /$ Graphene heterostructures. ACS Nano 2013, 7 (4), 3246-3252.

[63] Hong, Augustin J., Song, Emil B., Yu, Hyung Sk., Matthew, J., Allen, J Kim., Fowler, J. D., Wassei, Jonathan K. , Park, Youngju., Wang, Yong., Zou, Jin., Kaner, R. B., Weiller, B. H., Wang, L., Graphene flash memory. ACS Nano 2011, 5 (10), 7812-7817. 